Bull. Korean Math. Soc. 51 (2014), No. 1, pp. 1-11

http://dx.doi.org/10.4134/BKMS.2014.51.1.001

\title{
A LYAPUNOV CHARACTERIZATION \\ OF ASYMPTOTIC CONTROLLABILITY FOR NONLINEAR SWITCHED SYSTEMS
}

\author{
YANLING Wang AND Ailing QI
}

\begin{abstract}
In this paper, we show that general nonlinear switched systems are asymptotically controllable if and only if there exist controlLyapunov functions for their relaxation systems. If the switching signal is dependent on the time, then the control-Lyapunov functions are continuous. And if the switching signal is dependent on the state, then the control-Lyapunov functions are $C^{1}$-smooth. We obtain the results from the viewpoint of control system theory. Our approach is based on the relaxation theorems of differential inclusions and the classic Lyapunov characterization.
\end{abstract}

\section{Introduction}

The problem of Lyapunov characterizations of asymptotic behavior is of particular interest in the design and analysis of robust feedback controls. It can be traced back to the pioneering work of Lyapunov [9], and has been extensively studied since 1950s'. As for the literature on asymptotic stability analysis of switched systems, despite the fact that it is vast and growing, the Lyapunov characterization method with the relaxation theorems of differential inclusions is still one of the very few starting points for nonlinear switched systems.

By a nonlinear switched system, we mean a hybrid dynamical system that is composed of a family of continuous-time subsystems and a rule orchestrating the switching signal between the subsystems (see [2]). The controllability of nonlinear switched systems has been discussed for a long time. A popular technique for this research relies on Lie algebraic methods (see $[1,7,10]$ ). In this paper, we consider the asymptotic controllability of nonlinear switched systems by using the Lyapunov characterization with the relaxation theorems

Received May 1, 2011; Revised April 29, 2013.

2010 Mathematics Subject Classification. 34D20, 93B05, 93D05, 93D20.

Key words and phrases. switched systems, control systems, asymptotically controllable, control-Lyapunov function, differential inclusions.

Supported by NNSF of China(10771159), NSF of Tianjin(09JCYBJC01800) and the Foundation Research Funds for the Central Universities through grant No. ZXH2012B003 and ZXH2012K002. 
of differential inclusions. The problem can be described by the form

$$
\dot{x}=f_{\sigma}(x), \quad x \in \mathbb{R}^{n}, \quad \sigma \in P,
$$

where $P=\{1,2, \ldots, m\}$, and the function $f_{\sigma}$ is Lipschitz with $f_{\sigma}(0)=0$ for each $\sigma \in P$. As a matter of fact, the switched system (1.1) can be recast as

$$
\dot{x}=\sum_{i=1}^{m} f_{i}(x) u_{i},
$$

where the admissible controls are of the form $u_{k}=1, u_{i}=0 \forall i \neq k$ (this corresponds to $\sigma=k$ ). So the switching signal $\sigma$ can be seen as an input control, thus the switched system (1.1) can be described as a control system with a piecewise constant control

$$
\dot{x}=u \cdot f(x), \quad x \in \mathbb{R}^{n},
$$

where the control input $u:[0,+\infty) \rightarrow \mathbb{U}$ is a piecewise constant function and $\mathbb{U}=\left\{e_{1}, e_{2}, \ldots, e_{m}\right\}$ is the standard orthonormal basis of $\mathbb{R}^{m}$. The function $f=\left(f_{1}, f_{2}, \ldots, f_{m}\right)$ and every $f_{i}: \mathbb{R}^{n} \rightarrow \mathbb{R}^{n}(i=1,2, \ldots, m)$ are Lipschitz. In this way, the switched system (1.1) is equivalent to the control system (1.3), thus we may research properties of the nonlinear switched systems within the framework of general control systems.

Sontag proved in [11] that, a control system in $\mathbb{R}^{n}$ is asymptotically controllable to the origin if and only if there exists a positive definite continuous function of the states whose derivative can be made negative by appropriate choices of controls. Later on, the result of [11] was improved in [12]. Subsequently, Clark, Ledyaev and Stern showed in [6] that some of these results can be generalized to differential inclusions. In fact, it was proved in [6] that, differential inclusions corresponding to upper semicontinuous multifunctions are strongly asymptotically stable if and only if there exists a smooth Lyapunov function. Almost meanwhile, Ledyaev and Sontag proved in [8] that, the existence of a smooth uniform control Lyapunov function for general nonlinear control system (2.1) if and only if there exists a robustly stabilizing feedback.

Our purpose is to apply the results in the reference [8] and [12] to this paper by founding a relationship of solutions between switched systems and control systems, so the control $u$ is required to be measurable and essentially bounded, then we take account of the following control system corresponding to the switched system $(1.3)$

$$
\dot{x}=u \cdot f(x), \quad x \in \mathbb{R}^{n},
$$

where $u \in L^{\infty}([0,+\infty) ; \mathbb{U})$.

However, there is still a difficulty that the control set $\mathbb{U}$ is not convex, then we convexify the control set by setting $u(t) \in \overline{c o} \mathbb{U}$, where $\overline{c o} \mathbb{U}$ is the closed convex hull of $\mathbb{U}$. So we obtain the following control system

$$
\dot{x}=u \cdot f(x), \quad x \in \mathbb{R}^{n},
$$

where $u \in L^{\infty}([0,+\infty) ; \overline{c o} \mathbb{U})$. 
Despite a finite number of switching signals are extended into an infinite number of input controls, the solution set of switched systems is dense in solution set of its relaxation systems. Actually, we will prove in Section 3 that every solution of the control system (1.5) can be approximated by one solution of the control system (1.3), which implies that the asymptotic controllability is equivalent between the control system (1.3) and (1.5).

It is well known that stability for arbitrary switching is a basic problem regarding stability and design of switched systems. Because spaces of switching signals do not have any completeness properties, switching is in principle very hard to study. The results that we present here allow us achieve a simplification that is, switching signals can be understood in terms of arbitrary Lebesguemeasurable controls. we wish to emphasize that the results that we present not only to extend some of those in [8] and [12], but get a better comprehension of the structure of the approximation of solutions of this class of nonlinear switched systems.

This paper is organized as follows. In Section 2, we provide some basic definitions and auxiliary results which will be required in this paper. In Section 3, we prove the Lyapunov characterization for asymptotic controllability of the control system (1.3). Finally, in Section 4, our conclusions are given.

\section{Basic knowledge}

Let us start by considering general nonlinear control systems of the type

$$
\dot{x}=f(x, u), \quad x \in \mathbb{R}^{n},
$$

where the function $f$ is locally Lipschitz, the control $u$ is a measurable and essentially bounded function with taking value in a locally compact and convex metric space $U$.

For general nonlinear control systems (2.1), there have been rich results. Conclusions related to a Lyapunov characterization of asymptotic controllability for open loop systems are made in [12] and [11], and a Lyapunov characterization of feedback stability for closed loop systems or differential inclusions are made in [10] and [6].

Definition 2.1 ([11]). We say that the control system (2.1) is asymptotically controllable if the following properties hold:

(i) For each $x \in \mathbb{R}^{n}$ there exists a control $u \in U$ such that the responding solution $x(t)$ from the initial state $x$ is defined for all $t \geq 0$ and $x(t) \rightarrow 0$ as $t \rightarrow \infty$.

(ii) For each $\varepsilon>0$ there exists a $\delta>0$ such that for any state $x \in \mathbb{R}^{n}$ with $|x|<\delta$ there is a control $u \in U$ as in (i) such that $|x(t)| \leq \varepsilon$ for all $t \geq 0$.

(iii) There exist positive $\eta$ and $k$ such that, if the state $x$ in (ii) satisfies also $|x|<\eta$, then the control can be chosen with $|u| \leq k$.

A function $V: \mathbb{R}^{n} \rightarrow[0,+\infty)$ is positive definite if

$$
V(0)=0 \text { and } V(x)>0 \text { for } \forall x \neq 0,
$$


and $V$ is proper if

$$
V(x) \rightarrow \infty \text { as }|x| \rightarrow \infty .
$$

Definition $2.2([12])$. For a function $V: \mathbb{R}^{n} \rightarrow[0,+\infty)$, a state $x \in \mathbb{R}^{n}$ and a direct $v \in \mathbb{R}^{n}$, the upper contingent derivative of $V$ in the direction $v$ at $x$ is defined as

$$
D_{v}^{+} V(x):=\liminf _{t \rightarrow 0^{+}, w \rightarrow v} \frac{1}{t}[V(x+t w)-V(x)] .
$$

The upper contingent derivative is widely studied in set-valued analysis and differential inclusion $[3,4,5]$. Observe that if $V$ is Lipschitz continuous, then this definition coincides with the classical Dini derivative, that is

$$
D V(x)(v):=\liminf _{t \rightarrow 0^{+}} \frac{1}{t}[V(x+t v)-V(x)] .
$$

Naturally, if $V$ is differentiable at $x$, then $D_{v}^{+} V(x)$ is the usual directional derivative $\langle\nabla V, v\rangle$.

Definition 2.3 ([12]). A continuous control-Lyapunov function for open loop system $(2.1)$ is a function $V: \mathbb{R}^{n} \rightarrow[0,+\infty)$ which is continuous, positive definite and proper such that there exists a continuous positive definite function $W: \mathbb{R}^{n} \rightarrow[0,+\infty)$ with the property that for each $x \in \mathbb{R}^{n}$ there is an $u \in U$ such that

$$
D_{f(x, u)}^{+} V(x) \leq-W(x)
$$

Definition 2.4 ([8]). A $C^{1}$-smooth control-Lyapunov function for closed loop system (2.1) is a function $V: \mathbb{R}^{n} \rightarrow[0,+\infty)$ which is $C^{1}$, positive definite and proper such that there exists a continuous positive definite function $W: \mathbb{R}^{n} \rightarrow$ $[0,+\infty)$ with the property that for each $x \in \mathbb{R}^{n}$ there is an $u=k(x) \in U$ such that

$$
\langle\nabla V(x), f(x, k(x))\rangle \leq-W(x) .
$$

When the switching signal is dependent on the time, the corresponding control system is an open loop system. In reference [12], E. D. Sontag and H. J. Sussmann give a continuous control-Lyapunov function characterization of asymptotic controllability when the control system (2.1) is an open loop system.

Lemma 2.5. The control system (2.1) is asymptotically controllable if and only if there exists a continuous control-Lyapunov function.

When the switching signal is dependent on the state, the corresponding control system is a closed loop system. The feedback stabilization problem is that of finding a feedback control $k: \mathbb{R}^{n} \rightarrow U$ such that the origin in $\mathbb{R}^{n}$ is asymptotically stable with respect to the trajectories of the closed loop system

$$
\dot{x}=f(x, k(x)) \text {. }
$$


In general, one of the most important reasons for using feedback control lies in feedback's robustness properties. By robustness we mean that for the perturbed closed loop system

$$
\dot{x}=f(x, k(x+e(t)))+w(t),
$$

the feedback $k$ drives the state of the system to a small neighborhood of the origin, even in the presence of measurement error $e(\cdot)$ (arbitrary bounded function $e:[0,+\infty) \rightarrow \mathbb{R}^{n}$ ) and external disturbance $w(\cdot)$ (measurable essentially bounded function $\left.w:[0,+\infty) \rightarrow \mathbb{R}^{n}\right)$.

Definition 2.6 $([8])$. The feedback $u=k(x)$ is said to be robustly stabilizing if for any $0<r<R$ there exist positive $T=T(r, R), \eta=\eta(r, R)$ and $M(R)$ such that, for any measurement error $e(\cdot)$ and external disturbances $w(\cdot)$ for which

$$
|e(t)| \leq \eta, \quad \forall t \geq 0 ; \quad\|w(\cdot)\|_{\infty} \leq \eta
$$

and every solution $x(\cdot)$ of the perturbed closed loop system with $|x(0)| \leq R$ does not blow up and satisfies the following relations:

1. (uniform attractivity)

$$
|x(t)| \leq r, \quad \forall t \geq T
$$

2. (bounded overshoot)

$$
|x(t)| \leq M(R), \quad \forall t \geq 0,
$$

3. (Lyapunov stability)

$$
\lim _{R \rightarrow 0} M(R)=0 .
$$

In reference [8], Y. S. Ledyaev and E. D. Sontag give a $C^{1}$-smooth controlLyapunov function characterization of robustly asymptotic controllability when the control system (2.1) is a closed loop system. The main result is the following lemma.

Lemma 2.7. The control system (2.1) admits a robustly stabilizing feedback if and only if there exists a $C^{1}$-smooth control-Lyapunov function for it.

Note that in this paper the function $f$ in the control system (2.1) is Lipschitz, so it is obvious that the existence of a robustly stabilizing feedback for the closed loop system is equivalent to the existence of a robustly asymptotically controllable feedback for it.

Upon defining the multifunction

$$
F(x)=f(x, U)=\bigcup_{u \in U} f(x, u),
$$

the control system (2.1) becomes a specially parametric differential inclusion of the form

$$
\dot{x} \in F(x), \quad x \in \mathbb{R}^{n},
$$


where $F$ is a set-valued function whose values are subsets of $\mathbb{R}^{n}$. As usual, a solution of (2.2) on an interval $[a, b]$ is an absolutely continuous function such that (2.2) holds a.e. on $[a, b]$.

We denote by

$$
B(K, \eta):=\left\{x \in \mathbb{R}^{n} \mid d(x, K) \leq \eta\right\}
$$

the ball of radius $\eta$ around the subset $K \subset \mathbb{R}^{n}$, and

$$
d_{H}(A, K):=\sup _{x \in A} d(x, K)
$$

is called the Hausdorff semidistance of subsets $A$ and $K$ in $\mathbb{R}^{n}$, where $d(x, K)=$ $\inf _{y \in K} d(x, y)$. It is clear that

$$
d_{H}(A, K) \leq \varepsilon \quad \text { if and only if } \quad A \subset B(K, \varepsilon) .
$$

Definition $2.8([5])$. Let $F: \mathbb{R}^{n} \rightarrow \mathbb{R}^{n}$ be a set-valued map. We say that $F$ is Lipchitz if for any $x, x^{\prime} \in \mathbb{R}^{n}$, there exists $L \geq 0$ such that

$$
F(x) \subset B\left(F\left(x^{\prime}\right), L d\left(x, x^{\prime}\right)\right) .
$$

Relaxation Theorem states that the solution set of an initial value problem for a Lipschitz compact valued differential inclusion $\dot{x} \in F(x)$ is dense in the solution set of the same initial value problem for the corresponding relaxation inclusion $\dot{x} \in \overline{c o} F(x)$. We state now Relaxation Theorem which is used in the proof of Lemma 3.5.

Theorem $2.9([4])$. Let $F$, from $Q=\left\{x \in \mathbb{R}^{n}|| x-x_{0} \mid \leq b\right\}$ into the compact subsets of $\mathbb{R}^{n}$, be a Lipschitz set-valued map. Set $I=[-T,+T]$ and let $x: I \rightarrow$ $Q$ be a solution to

$$
\dot{x}(t) \in \overline{c o}(F(x(t))), \quad x(0)=x_{0}
$$

such that, for $t \in I,\left\|x(t)-x_{0}\right\| \leq b$. Then for every positive $\varepsilon$, there exists $y: I \rightarrow Q$, a solution to

$$
\dot{y}(t) \in F(y(t)), \quad y(0)=x_{0}
$$

such that

$$
|y(t)-x(t)| \leq \varepsilon, \quad \forall t \in I
$$

\section{Main results}

We are now ready to state our main results.

Theorem 3.1. If the switching signal is dependent on the time, then the control system (1.3) is asymptotically controllable if and only if the control system (1.5) admits a continuous control-Lyapunov function $V: \mathbb{R}^{n} \rightarrow[0,+\infty)$, for each $x \in \mathbb{R}^{n}$ there is a $v \in \overline{c o}\left(f_{i}\right)$ such that

$$
D_{v}^{+} V(x) \leq-W(x) \quad \forall x \in \mathbb{X}, \quad x \neq 0 .
$$


Theorem 3.2. If the switching signal is dependent on the state, then the control system (1.3) exists a robustly asymptotically controllable feedback if and only if the closed loop system (1.5) admits a $C^{1}$-smooth control-Lyapunov function $V: \mathbb{R}^{n} \rightarrow[0,+\infty)$ for each $x \in \mathbb{R}^{n}$ there is a $v=k(x) \cdot f(x) \in \overline{c o}\left(f_{i}\right)$ such that

$$
\langle\nabla V(x), v\rangle \leq-W(x), \quad \forall x \in \mathbb{X}, \quad x \neq 0 .
$$

To prove Theorems 3.1 and 3.2, we first analyze the relation between the solutions of the control system (1.3), (1.4) and (1.5) and prove it.

Lemma 3.3. Assume that $y(t)$ is a solution of the control system (1.4) defined on $[0,+\infty)$. Then for any $\varepsilon>0$, there exists a solution $x(t)$ of the control system (1.3) with $x(0)=y(0)$ such that

$$
|x(t)-y(t)| \leq \varepsilon, \quad \forall t \in[0,+\infty) .
$$

Proof. Assume that $y(t)$ is a solution of the control system (1.4) defined on $[0,+\infty)$ with $y(0)=\xi_{0}$ and the corresponding control $u \in L^{\infty}([0,+\infty) ; \mathbb{U})$. For any $\varepsilon>0$, take

$$
\Omega_{1}=\left\{t \in[0,+\infty) \mid u(t)=e_{1}\right\},
$$

then there exists a sequence of intervals $I_{1_{k}}, k=1,2, \ldots$ which is not intersected with each other such that $m\left(\bigcup I_{1_{k}} \backslash \Omega_{1}\right) \leq \varepsilon / m$. Let

$$
v_{1}(t)= \begin{cases}e_{1}, & t \in \bigcup I_{1_{k}}, \\ 0, & \text { otherwise. }\end{cases}
$$

We have $\int_{\Omega_{1}}\left|v_{1}(t)-u(t)\right| d t \leq \varepsilon / m$.

In the same way, take

$$
\Omega_{2}=\left\{t \in[0,+\infty) \mid u(t)=e_{2}\right\},
$$

then there exists a sequence of intervals $I_{2 k}, k=1,2, \ldots$ which is not intersected with each other such that $m\left(\bigcup I_{2_{k}} \backslash \Omega_{2}\right) \leq \varepsilon / m$. Let

$$
v_{2}(t)= \begin{cases}e_{2}, & t \in \bigcup I_{2_{k}} \\ 0, & \text { otherwise. }\end{cases}
$$

We have $\int_{\Omega_{2}}\left|v_{2}(t)-u(t)\right| d t \leq \varepsilon / m$.

We proceed in this way until we take

$$
\Omega_{m}=\left\{t \in[0,+\infty) \mid u(t)=e_{m}\right\},
$$

then there exists a sequence of intervals $I_{m_{k}}, k=1,2, \ldots$ which is not intersected with each other such that $m\left(\bigcup I_{m_{k}} \backslash \Omega_{m}\right) \leq \varepsilon / m$. Let

$$
v_{m}(t)= \begin{cases}e_{m}, & t \in \bigcup I_{m_{k}}, \\ 0, & \text { otherwise. }\end{cases}
$$

We have $\int_{\Omega_{m}}\left|v_{m}(t)-u(t)\right| d t \leq \varepsilon / m$.

Define

$$
v(t)=v_{1}(t)+v_{2}(t)+\cdots+v_{m}(t)
$$


If the intersection of intervals $I_{h_{i}}$ and $I_{l_{j}}$ is not empty, here $1 \leq h \leq m$, $1 \leq l \leq m, h \neq l$ and $i, j=1,2, \ldots$, then we assume $h<l$ as well and redefine

$$
v(t)=e_{h}, \quad \forall t \in I_{h_{i}} \bigcap I_{l_{j}} .
$$

Obviously, $v:[0,+\infty) \rightarrow \mathbb{U}$ is piecewise constant.

Let $I=\bigcup_{j=1}^{m} \bigcup_{k=1}^{\infty} I_{j_{k}}$, then $m([0,+\infty) \backslash I) \leq \varepsilon, I \bigcup I_{0}=[0,+\infty)$ with $I_{0}$ is a set of measure zero and

$$
\int_{I}|v(t)-u(t)| d t \leq \varepsilon
$$

We conclude that there exists a solution $x(t)$ of the control system (1.3) corresponding control $v$ with $x(0)=\xi_{0}$. We next prove $|x(t)-y(t)| \leq \varepsilon$ for any $t \in[0,+\infty)$ by selecting $I$ such that

$$
\operatorname{diam}(I)<1 / \sup _{t \in I}|f(x(t))|
$$

here $\operatorname{diam}(I)=\max _{1 \leq j \leq m} \operatorname{diam}\left(I_{j_{k}}\right)$.

Since $x(t)$ is a solution of the control system (1.3), we can describe it with the integral form that is

$$
x(t)=x(0)+\int_{0}^{t} u(s) \cdot f(x(s)) d s, \quad t \in[0,+\infty) .
$$

Similarly, the integral form of $y(t)$ is

$$
y(t)=y(0)+\int_{0}^{t} v(s) \cdot f(x(s)) d s, \quad t \in[0,+\infty) .
$$

Then for any $t \in[0,+\infty)$, we have

$$
\begin{aligned}
|x(t)-y(t)| & =\left|\int_{0}^{t} u(s) \cdot f(x(s)) d s-\int_{0}^{t} v(s) \cdot f(x(s)) d s\right| \\
& \leq \int_{I}|u(s)-v(s)||f(x(s))| d s \\
& \leq \operatorname{diam}(I) \cdot \sup _{t \in I}|f(x(t))| \cdot \varepsilon \\
& =\varepsilon
\end{aligned}
$$

Remark 3.4. It suffices to derive the conclusion that the control system (1.4) is asymptotically controllable if and only if the control system (1.3) is asymptotically controllable by the definition of asymptotic controllability and Lemma 3.3 .

Lemma 3.5. Pick any $T>0$. Let $z(t)$ be any solution of the control system (1.5) defined on $[0, T]$, then, for any $\varepsilon>0$, there exists a solution $y(t)$ of the control system (1.4) with $y(0)=z(0)$ such that

$$
|y(t)-z(t)| \leq \varepsilon, \quad \forall t \in[0, T] .
$$


Proof. For any $x \in \mathbb{R}^{n}$, let

$$
G(x)=\left\{u \cdot f(x) \mid u \in L^{\infty}([0,+\infty) ; \mathbb{U})\right\},
$$

then the solution set of differential inclusion

$$
\dot{x} \in G(x), \quad x \in \mathbb{R}^{n}
$$

is equal to the solution set of the control system (1.4).

In the similar way, for any $x \in \mathbb{R}^{n}$, let

$$
H(x)=\left\{u \cdot f(x) \mid u \in L^{\infty}([0,+\infty) ; \overline{c o} \mathbb{U})\right\},
$$

then the solution set of differential inclusion

$$
\dot{x} \in H(x), \quad x \in \mathbb{R}^{n}
$$

is equal to the solution set of the control system (1.5).

It is easy to conclude that $H(x)=\overline{c o} G(x)$ for any $x \in \mathbb{R}^{n}$ by the definitions of set-value function $G$ and $H$.

Now we proof that the set-valued function $G$ is Lipschitz. Indeed, based on the fact that the function $f$ is Lipschitz, for any $x, x^{\prime} \in \mathbb{R}^{n}$, there exists $L>0$ such that

$$
d\left(f(x), f\left(x^{\prime}\right)\right) \leq L \quad d\left(x, x^{\prime}\right)
$$

then

$$
\begin{aligned}
d_{H}\left(G(x), G\left(x^{\prime}\right)\right) & =\sup _{u \in \mathbb{U}} d\left(u \cdot f(x), G\left(x^{\prime}\right)\right) \\
& =\sup _{u \in \mathbb{U}} \inf _{v \in \mathbb{U}} d\left(u \cdot f(x), v \cdot f\left(x^{\prime}\right)\right) \\
& \leq \sup _{u \in \mathbb{U}} d\left(u \cdot f(x), u \cdot f\left(x^{\prime}\right)\right) \\
& \leq \sup _{u \in \mathbb{U}}|u| d\left(f(x), f\left(x^{\prime}\right)\right) \\
& \leq L d\left(x, x^{\prime}\right),
\end{aligned}
$$

so we have

$$
G(x) \subset B\left(G\left(x^{\prime}\right), L d\left(x, x^{\prime}\right)\right), \quad \forall x, x^{\prime} \in \mathbb{R}^{n} .
$$

It is obvious that for any given $x \in \mathbb{R}^{n}, G(x)$ is a compact subset of $\mathbb{R}^{n}$.

This completes the proof of the lemma from the Relaxation Theorem 2.9.

Lemma 3.6. The control system (1.5) is asymptotically controllable if and only if the control system (1.4) is asymptotically controllable.

Proof. The sufficiency is clear, so in the following we will only prove the necessary part.

Assume that the control system (1.5) is asymptotically controllable, then for every initial state $\xi_{0} \in \mathbb{R}^{n}$ there is a control $u_{1} \in L^{\infty}([0,+\infty) ; \overline{c o} \mathbb{U})$ and a resulting solution $z_{1}(t)$ that drives $\xi_{0}$ to 0 . From item (i) in the definition of asymptotic controllability, there is a $T_{1}>0$ such that

$$
\left|z_{1}(t)\right| \leq \varepsilon / 2, \quad \forall t \geq T_{1} .
$$


By virtue of the above Lemma 3.5, there is a solution $y_{1}(t)$ of the control system (1.4) and a control $v_{1} \in L^{\infty}([0,+\infty) ; \mathbb{U})$ such that $y_{1}(0)=\xi_{0}$ and

$$
\left|y_{1}(t)-z_{1}(t)\right| \leq \varepsilon / 2, \quad t \in\left[0, T_{1}\right] .
$$

Note $y_{1}\left(T_{1}\right)=\xi_{1}$, we have

$$
\left|\xi_{1}\right| \leq\left|y_{1}\left(T_{1}\right)-z_{1}\left(T_{1}\right)\right|+\left|z_{1}\left(T_{1}\right)\right| \leq \varepsilon
$$

For each $\xi_{1} \in \mathbb{R}^{n}$ there is a control $u_{2} \in L^{\infty}([0,+\infty) ; \overline{c o} \mathbb{U})$ and a resulting solution $z_{2}(t)$ with $z_{2}(0)=\xi_{1}$ that drives $\xi_{1}$ to 0 . It follows by Definition 2.1 that there is a $T_{2}>T_{1}$ such that

$$
\left|z_{2}\left(t-T_{1}\right)\right|<\varepsilon / 4, \quad \forall t \geq T_{2} .
$$

Due to the above Lemma 3.5, we obtain that there is a solution $y_{2}(t)$ of the control system (1.4) and a control $v_{2} \in L^{\infty}([0,+\infty) ; \mathbb{U})$ such that $y_{2}(0)=\xi_{1}$ and

Similarly, note $y_{n}\left(T_{n}\right)=\xi_{n}$, we then have

$$
\left|y_{2}\left(t-T_{1}\right)-z_{2}\left(t-T_{1}\right)\right| \leq \varepsilon / 4, \quad t \in\left[T_{1}, T_{2}\right]
$$

$$
\left|\xi_{n}\right| \leq\left|y_{n}\left(T_{n}\right)-z_{n}\left(T_{n}\right)\right|+\left|z_{n}\left(T_{n}\right)\right| \leq \varepsilon / n .
$$

For each $\xi_{n} \in \mathbb{R}^{n}$ there is a control $u_{n+1} \in L^{\infty}([0,+\infty) ; \overline{c o} \mathbb{U})$ and a resulting solution $z_{n+1}(t)$ with $z_{n+1}(0)=\xi_{n}$ that drives $\xi_{n}$ to 0 , also by Definition 2.1 there is a $T_{n+1}>T_{n}$ such that

$$
\left|z_{n+1}\left(t-T_{n}\right)\right|<\varepsilon /[2(n+1)], \quad \forall t \geq T_{n+1} .
$$

We apply Lemma 3.5 and obtain that there is a solution $y_{n+1}(t)$ of the control system (1.4) and a control $v_{n+1} \in L^{\infty}\left([0,+\infty) ; \mathbb{R}^{n}\right)$ such that $y_{n+1}(0)=\xi_{n}$ and

$$
\left|y_{n+1}\left(t-T_{n}\right)-z_{n+1}\left(t-T_{n}\right)\right| \leq \varepsilon /[2(n+1)], \quad t \in\left[T_{n}, T_{n+1}\right] .
$$

Now we obtain a control $v \in L^{\infty}([0,+\infty) ; \mathbb{U})$ immediately by setting

$$
v(t)=v_{n}(t), \quad t \in\left[T_{n-1}, T_{n}\right], n=1,2, \ldots,
$$

and a corresponding solution $y(t)$ of the control system (1.4) by defining

$$
y(t)=y_{n}(t), \quad t \in\left[T_{n-1}, T_{n}\right], \quad n=1,2, \ldots,
$$

with $T_{0}=0, y(0)=\xi_{0}$ and $y(t) \rightarrow 0$ as $t \rightarrow \infty$ which implies that the control system (1.4) is asymptotically controllable. This completes the proof of the desired result.

Remark 3.7. It follows from Remark 3.4 and Lemma 3.6 that the control system (1.5) is asymptotically controllable if and only if the control system (1.3) is asymptotically controllable.

Proof of Theorem 3.1 and Theorem 3.2. By the results of Lemmas 2.5 and 2.7 and by Remark 3.7 we can get the conclusions of Theorem 3.1 and Theorem 3.2 immediately. 


\section{Conclusions}

In this paper, we have established a necessary and sufficient condition of stabilization of a certain class of nonlinear switched systems. The proof of the theorems are greatly facilitated by establishing an association between the switched systems and the control systems, and by using the relaxation theorems of differential inclusions and the control-Lyapunov characterization.

Acknowledgments. The authors express their sincere thanks to Professor Desheng Li for his instructions and many invaluable suggestions. The authors are also grateful to the anonymous referees for their careful reading of the manuscript and helpful suggestions. Furthermore, the authors thank the reviewer for correcting errors and suggesting improvements in the organization of this paper.

\section{References}

[1] A. A. Agrachev and D. Liberzon, Lie-algebraic stability criteria for switched systems, SIAM J. Control Optim. 40 (2001), no. 1, 253-269.

[2] P. J. Antsaklis, J. A. Stiver, M. D. Lemmon et al., Hybrid Systems, Vol. 736 of Lecture Notes in Computer Science, 366-392, Heidelberg, Springer, 1993.

[3] J. P. Aubin, Viability Theory, Birkhäuser Boston, Inc., Boston, MA, 1991.

[4] J. P. Aubin and A. Cellina, Differential Inclusions, Springer-Verlag, Berlin, 1984.

[5] J. P. Aubin and H. Frankowska, Set-Valued Analysis, Springer-Verlag, Birkhauser Boston Basel Berlin, 1990.

[6] F. H. Clarke, Y. S. Ledyaev, and R. J. Stern, Asymptotic stability and smooth Lyapunov functions, J. Differential Equations 149 (1998), no. 1, 69-114.

[7] R. Gilmore, Lie Groups, Lie Algebras and Some of Their Applications, John Wiley, New York, 1994.

[8] Y. S. Ledyaev and E. D. Sontag, A Lyapunov characterization of robust stabilization, Nonlinear Anal. 37 (1999), no. 7, 813-840.

[9] A. M. Lyapunov, The general problem of the stability of motion, Math. Soc. Kharkov, 1892 (Russian); English Translation: Internat. J. Control 55 (1992), 531-773.

[10] P. J. Olver, Applications of Lie Groups to Differential Equations, 2nd ed. New York, Springer-Verlag, 1993.

[11] E. D. Sontag, A Lyapunov-like characterization of asymptotic controllability, SIAM J. Control Optim. 21 (1983), no. 3, 462-471.

[12] E. D. Sontag and H. J. Sussmann, Nonsmooth control Lyapunov functions, Proc. IEEE Conf. Decision and Control, New Orleans, Dec. 1995, IEEE Publications, 2799-2805, 1995.

YANLING WANG

Department of Mathematics

TIANJIN UnIVERSITY

Tianjin 300072, P. R. China

E-mail address: wangyl@tju.edu.cn

AiLing QI

College of Science

Civil Aviation University of China

Tianjin 300300, P. R. China

E-mail address: qiailing2010@gmail.com 